\title{
The role of cytokines in degenerative spine disorders
}

Original Paper

\author{
Sutovsky J., ${ }^{1}$ Kocmalova M. ${ }^{2,3 凶}$ Benco M., ${ }^{1}$ Kazimierova I., Pappova L., ${ }^{2,3}$ Frano A., ${ }^{4}$ Sutovska M., ${ }^{2,3}$ \\ ${ }^{1}$ Comenius University in Bratislava, Jessenius Faculty of \\ Medicine in Martin, Martin University Hospital, \\ Neurosurgery Clinic, Martin, Slovak Republic \\ ${ }^{2}$ Comenius University in Bratislava, \\ Jessenius Faculty of Medicine in Martin \\ Biomedical Centre Martin, Martin, Slovak Republic \\ ${ }^{3}$ Comenius University in Bratislava, \\ Jessenius Faculty of Medicine in Martin \\ Department of Pharmacology, Martin, Slovak Republic \\ ${ }^{4}$ Charles University Prague, 1st Medical Faculty, \\ The Hospital Na Bulovce, The Ortopedic Clinic, \\ Prague, Czech Republic
}

Received 21 February, 2017, accepted 11 April, 2017

Abstract Background: Degenerative spine disorders (DSD) are the most frequent reason of morbidity in adults. Commonly DSD includes degenerative disorders of intervertebral discs (IVDs), spinal stenosis and degenerative spondylolisthesis (SL). There is increasing evidence about significant role of cytokines in DSD pathogenesis, symptomathology and progression, but their protective levels remain still unknown.

Material and Methods: The aim of presented study was to provide quantitative and qualitative analysis of cytokine, chemokine and growth factors levels in individual parts of IVDs - annulus fibrosus (AF) and nucleus pulposus (NP) - separately and in facet joints (FJ) subchondral bone of patients with DSD and in controls - healthy subjects during a multiorgan procurement procedure. Bio-Plex ${ }^{\circledast}$ assay was used to measure concentrations of 27 different cytokines in tissue of patients with DSD. Their concentrations in tissues of healthy subjects during a multiorgan procurement procedure represented protective levels.

Results: The Bio-Plex ${ }^{\circledast}$ assay revealed significant differences between the patients suffered from degenerated and herniated IVDs and from lumbar SL and controls in cytokines, chemokines and growth factor profiles suggested that pro-inflammatory changes of both NP and AF were dominated in herniated IVDs, whereas the same tissue of lumbar SL patients exhibited much more complex changes in cytokine levels suggested o only ongoing inflammation (IL-6, IL-8, MCP-1, TNF-a), abut also antiinflammatory processes (IL-ra, IL-10) or connective tissue remodeling (PDGF-bb, IL-17, VEGF). The different mediators were found elevated in lumbar SL samples of subchondral FJ bone. These also confirmed ongoing inflammation, accelerated bone resorption and formation and increased fibroblasts activity in FJ bone.

Conclusion: The study supported the significant involvement of several cytokines, chemokines and growth factors in the pathogenesis of DSD. These cytokines should represent future potential targets for new biological treatment able to slow DSD progression as well as factor determining prognosis of DSD.

Keywords Degenerative lumbar spondylolisthesis - herniated intervertebral disc-cytokine levels-annulus fibrosus - nucleus pulposus

\section{INTRODUCTION}

The degenerative spine disorders (DSD) includes disorders of intervertebral discs (IVDs) and degenerative spondylolisthesis. DSD represents the most frequent reason of morbidity in adults and its incidence rises with age. The common clinical signs of DSD are pain and mobility impairment, as the consequence of spinal roots and spinal cord compression by herniated IVDs, spinal canal stenosis, osteoarthrosis and damage of facet joints (FJs) cartilage. The pain can appear without evident neuronal compression due to the local release of cytokines and chemokines. It has been recently appointed a significant role of cytokines, not only in pain mediation, but also in DSD progression (Wuertz and Haglund, 2013).

IVDs consist of annulus fibrosus (AF) and nucleus pulposus (NP). While collagen represents the main compound of AF, NP comprises proteoglycans and water. One of the early symptoms of IVD degeneration is reduced hydration of NP, followed by subsequent biochemical and structural changes that change the mechanic loading of spine (Risbud and Shapiro, 2014).

*E-mail: Michaela.Kocmalova@jfmed.uniba

(c) European Pharmaceutical Journal 
The microtraumatic changes appear in AF that initiate the local release of cytokines and chemokines, which trigger local inflammatory reaction and simultaneously regulate the anabolic and catabolic processes in IVDs. Typical feature of degenerated IVDs is the loss of physiological balance between matrix production and degradation, which can possibly result in protrusion or herniation of NP even in complete failure of IVD structure (Wuertz and Haglund, 2013; Risbud and Shapiro, 2014). Spondylolisthesis (SL) is defined as anterior slippage of cranial vertebra in relation to the adjacent vertebra. Although there are 5 types of SL: dysplastic, isthmic, traumatic, pathologic and degenerative. Degenerative SL, localized in lumbar spine, is by far the most frequent. It is generally accepted that degenerative SL occurs as the result of alterative inflammation and FJs subchondral bone remodelling(Wuertz and Haglund, 2013), or as a result of FJ overload at IVD failure.

The cytokines play an important regulatory and amplifying role under the physiological conditions as well as in IVD degeneration or in SL development (Wuertz and Haglund, 2013; Lee et al., 2013). The catabolic processes in IVD tissues are significantly accelerated by interleukin-1 (IL-1), IL-2, IL-4, IL-6, IL-8 and tumour necrosis factor-a (TNF-a) (Wuertz and Haglund, 2013; Risbud and Shapiro, 2014; Lee et al., 2013). Simultaneously, IL-1ß, IL-6, IL-8 and TNF-a stimulate synthesis of neuronal growth factor (NGF) and vascular endothelial growth factor (VEGF) that regulate the neuronal proliferation and neoangiogenesis in AF, NP and FJs subchondral bone, control bone remodelling and irritate nociceptors (Wuertz and Haglund, 2013; Lee et al., 2013; Raggatt and Partridge, 2010). Despite several recently published papers describing the changes incytokine levels in DSD, the role of cytokines in disease pathogenesis is not still completely explained. One of the reasons is that we still do not know their levels produced under the physiological conditions in healthy subjects, which have a protective character. Although several studies compared cytokine levels in the IVDs of operated patients with their levels in healthy cadaveric tissue samples, but donors at the time of collection were dead for more than 24 hours (Le Maitre et al., 2007). According to the limited stability of cytokines, it is not possible to exclude that such an increase in the patient's cytokine levels may have been caused due to secondary factors (Friebe and Volk, 2008). The works, for example, comparing the cytokine levels in patients with DSD and healthy subjects, are not presented in scientific literature. This study was designed to exclude the secondary distort of results. The control subjects are living organ donors and sample handling methods allow to maintain stable cytokine levels. The data obtained this way should significantly contribute to the clarification of cytokine and chemokine roles in the pathophysiology and symptomatology of DSD.

\section{MATERIAL AND METHODS}

The main aim of our study was to investigate the cytokines, chemokines and the profile of growth factors in the samples of $A F, N P$ and FJs bone from patients with $S L, A F$ and NP from patients with degenerated and herniated IVDs as well as from healthy donors.

All processes were approved by the Institutional Ethic Committee of the Jessenius Faculty of Medicine registered in the Institutional Review Board/Institutional Ethic Board Office (IRB 00005636) in accordance with the Slovakian and European legislation (decision No. EK 1209/2012). The patient's recruitment was performed by providing information sheets describing the study, and the patients provided written informed consent. A total of 10 patients (males, mean age of $48.6 \pm 14.1$ years), who had been operated on due to herniated lumbar intervertebral disc and 9 patients suffered from lower segment spondylolisthesis, Pfirrman's score 4-5 ( 2 males and 7 females; average age $50.7 \pm 10.2$ years) as verified on magnetic resonance images (MRI), were included in the study. The patients underwent primary spinal surgery at the Neurosurgery Clinic, JFM CU and University Hospital Martin. The patients did not have any severe systemic chronic disorders (rheumatological, endocrine, cardiovascular and oncologic) and were not administered any epidural drugs, such as analgesics, steroids or anesthetizing agents, during general anaesthesia and at least 12 week before the surgical treatment of spondylolisthesis. Intact FJ bone and IVD samples, used as a control group, were obtained from 6 adult males (average age $44.2 \pm 13.2$ years) during a multiorgan procurement procedure at the Transplant and Vascular Surgery Clinic, JFM CU, Martin University Hospital.

After removing the disc tissue, the nucleus pulposus (NP) and annulus fibrosus (AF) were separated by the surgeon. The subchondral bone tissue (B) was obtained from the adjacent FJ. Then all the collected tissue samples were immediately transported to the laboratory in sterile polypropylene storage tubes and kept frozen at $-80^{\circ} \mathrm{C}$.

Preparation and determination of proteins: Tissue was removed from IVD and facet joints bone (FJB) and lysed using a lysis kit (Bio-PlexTM Cell Lysis Kit, Bio-Rad, USA). After that the tissue was prepared using $3 \times 3 \mathrm{~mm}$ segments and washed using cell wash buffer. Tissue segments were transferred to a tube containing $500 \mu \mathrm{l}$ of lysis solution (40 $\mu$ l Factor 1, $20 \mu \mathrm{l}$ Factor 2 and $9.9 \mathrm{ml}$ Cell Lysis Buffer) incubated on ice prior to adding $40 \mu \mathrm{l}$ phenylmethylsulfonyl fluoride (PMSF). This mixture was homogenized (Homogenizator; Stuart SHM2, Germany) for 4 min at 4000 rotations per minute (RPM) stage 1. The homogenate was centrifuged for $4 \mathrm{~min}$ at $5000 \mathrm{RPM}$ $(2370 \mathrm{G})$, and the supernatant was collected and frozen at $-80^{\circ} \mathrm{C}$. Final homogenate of proteins were solubilized by the addition of $10 \%$ SDS solution to final concentration of SDS $5 \%$. Protein was measured by the method of Lowry et al. (1951) using bovine serum albumin as the standard.

The assessment of cytokine levels in tissue samples:The BioPlex $^{\circledast} 200$ System and Bio-PlexTM Human Cytokine Standard 27-Plex, Group I (Bio-Rad, Hercules, California, USA) were used to assess the cytokine levels. The method was previously described in detail by Franova et al. 2016. The total amount 
Table 1. Summary of the data obtained from the Bio-Plex ${ }^{\circledR}$ assay of nucleus pulposus (NP) tissue samples. The cytokine, chemokine and growth factors levels are expressed in $\mathrm{pg} / \mathrm{ml}$. ${ }^{*} p \leq 0.05$ and ${ }^{* *} p \leq 0.01$ Control samples vs. lumbar DS (Listhesis) or herniated discs (Hernia) specimens, \# $p \leq 0.05$ and \#\#p $\leq 0.01$ lumbar DS (Listhesis) vs herniated discs (Hernia) (one-way ANOVA with Bonferroni post-hoc test).

\begin{tabular}{|c|c|c|c|}
\hline & \multicolumn{3}{|c|}{ Nucleus pulposus (NP) } \\
\hline & Hernia & Listhesis & Control \\
\hline IL-1 $\beta$ & $4.35 \pm 0.45^{*}$ & $4.25 \pm 2.04$ & $2.11 \pm 0.81$ \\
\hline IL-1 ra & $34.50 \pm 3.85^{*}$ & $47.55 \pm 10.95^{*}$ & $86.22 \pm 13.41$ \\
\hline IL-6 & $795.36 \pm 140.71^{* * / \# \#}$ & $110.66 \pm 7.73^{*}$ & $41.64 \pm 15.43$ \\
\hline IL-8 & $90.26 \pm 57.56^{*}$ & $86.82 \pm 27.05^{*}$ & $22.79 \pm 1.75$ \\
\hline IL-10 & $52.21 \pm 1.23$ & $62.89 \pm 2.35^{\#}$ & $51.38 \pm 6.70$ \\
\hline IL-17 & $11.64 \pm 3.31$ & $14.61 \pm 2.57^{*}$ & $8.53 \pm 2.96$ \\
\hline Eotaxin & $562.87 \pm 142.77^{* *}$ & $476.90 \pm 214.06^{* *}$ & $36.70 \pm 13.78$ \\
\hline GM-CSF & $105.84 \pm 3.78$ & $145.81 \pm 46.72$ & $116.40 \pm 35.06$ \\
\hline IFN- $\boldsymbol{\gamma}$ & $51.61 \pm 6.75$ & $73.98 \pm 14.38^{*}$ & $55.66 \pm 12.57$ \\
\hline IP-10 & $7021.12 \pm 1642.28$ & $8272.19 \pm 3608.63^{\#}$ & $8482.56 \pm 3191.22$ \\
\hline MCP-1 & $262.85 \pm 50.99^{\#}$ & $137.52 \pm 44.11$ & $309.54 \pm 98.70$ \\
\hline RANTES & $246.85 \pm 157.22$ & $420.04 \pm 165.34^{\#}$ & $315.30 \pm 193.99$ \\
\hline TNF-a & $11.52 \pm 1.49^{*}$ & $19.24 \pm 2.47^{*} / \#$ & $5.16 \pm 0.88$ \\
\hline VEGF & $2277.02 \pm 205.05$ & $4328.05 \pm 44.32 * / \#$ & $2251.67 \pm 1142.14$ \\
\hline
\end{tabular}

of cytokines in individual samples was recalculated on their protein content.

Statistics:The results of the cytokine level assessment are presented as the mean \pm S.E.M. Data were evaluated with one-way analysis of variance (ANOVA) with the post hoc Bonferroni test using GraphPad Prism software version 6.01. Findings of $p \leq 0.05$ and lower were considered to be statistically significant.

\section{RESULTS AND DISCUSSION}

A total of 10 patients, who underwent lumbar discectomy due to herniated lumbar IVD and 9 patients suffering from lower segment SL who underwent neural structure decompression, IVD replacement and transpedicular stabilization, fulfilled inclusion criteria. The difference in age between patients and controls was found not to be statistically significant $(p=0.562)$. This study investigated the involvement of inflammatory mediators in DSD pathogenesis through the study of IVD and FJB tissues obtained from patients suffering from degenerated and herniated IVDs, lumbar SL and healthy controls during a multiorgan procurement procedure. Statistical analysis of the obtained data showed meaningful variations in the levels of several pro- and anti-inflammatory cytokines in the IVD and FJB tissue. The significantly elevated IL-1 $\beta$, IL-6, IL-8, eotaxin andTNF- $\alpha$ were measured in NP of herniated IVDs, whereas the same structure of patients with SL produced significantly higher levels IL-6, IL-8, IL-17, eotaxin, TNF- $a$ and vascular endothelial growth factor (VEGF). The production of antiinflammatory interleukin-1 receptor antagonist (IL-1ra) was suppressed in both DSD (Table 1).
External AF of herniated IVDs contained significantly higher levels of IL-6, IL-8, eotaxin and chemokine monocyte chemoattractant protein-1 (MCP-1) as well. The levels of eotaxin were not changed significantly in AF samples of patients with SL, but these samples contained higher concentration of granulocyte-macrophage colonystimulating factor (GM-CSF), TNF-a and VEGF in comparison to healthy subjects (Table 2 ).

Previously it was proposed that DSD involves complex interactions in the immune system to mediate inflammation, remodelling and degeneration of IVD. Several studies have already reported the elevated levels of IL-1 $\beta$, IL-6, IL-8, IL17 or TNF-a in patients with DSD (Le Maitre et al., 2007; Risbud and Shapiro, 2014). Despite similar pathogenesis, the inflammatory and immune activation profile exhibited by a degenerated IVD differs from that observed in lumbar SL. Our study, for the first time, demonstrated statistically significant differences between samples of patients suffering from degenerated and herniated IVDs and from lumbar SL, as it is apparent in Table 1 and 2. These variances together suggest that pro-inflammatory changes of both NP and AF dominated in herniated IVDs, whereas the same tissue of lumbar SL patients exhibited much more complex changes in cytokine levels suggested ongoing inflammation (IL-6, IL-8, MCP-1, TNF-a), anti-inflammatory processes (IL-ra, IL-10) or remodelling (PDGF-bb, IL-17, VEGF).

Furthermore, the Bio-Plex ${ }^{\circledast}$ assay revealed significantly elevated levels of the following cytokines, chemokines and growth factors in the FJB samples of patients suffering from lumbar SL compared to the controls: IL- 6 with $\mathrm{p} \leq 0.01$; IL-7, IL13, IFN- $\gamma$, TNF- $a$ and PDGF-bb with $p \leq 0.05$. These finding are 
Table 2. Summary of the data obtained from the Bio-Plex assay of annulus fibrosus (AF) tissue samples. For further explanation see also legend to Table 1.

\begin{tabular}{|c|c|c|c|}
\hline & \multicolumn{3}{|c|}{ Anulus fibrosus (AF) } \\
\hline & Hernia & Listhesis & Control \\
\hline PDGF-bb & $17.83 \pm 5.31$ & $37.82 \pm 4.43^{\#}$ & $41.09 \pm 22.26$ \\
\hline IL-1 ra & $31.16 \pm 4.02$ & $89.79 \pm 4.59^{\#}$ & $79.54 \pm 28.13$ \\
\hline IL-6 & $394.52 \pm 147.88^{* *}$ & $352.94 \pm 135.75^{* *}$ & $71.44 \pm 30.21$ \\
\hline IL-8 & $114.79 \pm 16.33^{*}$ & $89.20 \pm 14.08^{*}$ & $27.41 \pm 6.30$ \\
\hline IL-10 & $72.48 \pm 2.58$ & $150.00 \pm 92.68^{\#}$ & $69.17 \pm 28.53$ \\
\hline IL-17 & $8.82 \pm 0.80$ & $15.82 \pm 8.21^{\#}$ & $15.92 \pm 4.57$ \\
\hline Eotaxin & $298.70 \pm 55.56^{*} / \#$ & $101.81 \pm 52.14$ & $62.61 \pm 16.64$ \\
\hline GM-CSF & $135.18 \pm 35.35$ & $189.18 \pm 29.47^{*}$ & $109.42 \pm 19.64$ \\
\hline IP-10 & $5744.85 \pm 298.47$ & $11744.14 \pm 3026.78^{\#}$ & $6704.94 \pm 1450.79$ \\
\hline MCP-1 & $329.94 .46 \pm 42.65^{*}$ & $731.46 \pm 275.68^{*} / \#$ & $96.43 \pm 20.34$ \\
\hline RANTES & $1328.98 \pm 167.72$ & $2022.98 \pm 1263.72^{\#}$ & $1054.33 \pm 648.45$ \\
\hline TNF- $a$ & $10.47 \pm 1.37$ & $22.04 \pm 5.45 * / \#$ & $9.55 \pm 1.40$ \\
\hline VEGF & $2288.78 \pm 169.99$ & $5975.74 \pm 258.99 * / \#$ & $3021.25 \pm 296.80$ \\
\hline
\end{tabular}

consistent with Raggatt and Partridge (2010), who observed significantly higher concentrations of IFN $\gamma$, IL-6, IL-7 and IL-13 cytokines in synovial fluid of joints with cartilage defects and osteoarthritis than in healthy donors.

\section{CONCLUSIONS}

We can summarize that the study results confirmed significant involvement of the immune system, as represented by several cytokines, chemokines and growth factors, in the pathogenesis of DSD. The control samples, which were obtained during the multiorgan procurement procedures, represent the main strength of this study. This method should avoid any false findings due to cytokine level changes caused by cytokine degradation after the cessation of breathing, blood circulation and death. The main limitation of this study

\section{References}

[1] Franova S, Kazimierova I, Pappova L, Joskova M, Plank L, Sutovska $M$. Bronchodilatory, antitussive and anti-inflammatory effect of morin in the setting of experimentally induced allergic asthma. Journal of Pharmacy and Pharmacology. 2016;68(8):1064-1072.

[2] Friebe A, Volk HD. Stability of tumor necrosis factor alpha, interleukin 6, and interleukin 8 in blood samples of patients with systemic immune activation. Arch Pathol Lab Med. 2008;132:18021806.

[3] Lee AS, Ellman MB, Yan D, Kroin JS, Cole BJ, van Wijnen AJ, Im HJ. A current review of molecular mechanisms regarding osteoarthritis and pain. Gene 203;527:440-447.

[4] Le Maitre CL, Hoyland JA, Freemont AJ. Catabolic cytokine expression in degenerate and herniated human intervertebral discs: IL1ßand TNFa expression profile. Arthritis Res Ther. 2007; 9:R77. is the gender of the subjects included in the groups: only male subjects were included in control and herniated IVDs groups, while most of the lumbar SL patients were female. Only 2 female patients were of reproductive age, therefore there is low possibility that sex hormones interfere with cytokine levels. However, there is still much to be learned about the interactions among and the influence of many components that regulate the immune cascade in DSD. We believe that improving the knowledge and understanding of immune system participation in this disease could improve future therapeutic strategies.

\section{ACKNOWLEDGEMENTS}

This study was supported by the 'BioMed'project, which is cofinanced by EC sources and VEGA grant No. 1/0163/16.

[5] Lowry OH, Rosebrough NJ, Farr AL, Randall RJ. Protein measurement with the Folin phenol reagent. J Biol Chem. 1951;193:265275.

[6] Modic MT, Ross JS. Lumbar degenerative disk disease. Radiology 2007; 245:43-61.

[7] Raggatt LJ, Partridge NC. Cellular and molecular mechanisms of bone remodeling. J Biol Chem. 2010;285:25103-25108.

[8] Risbud MV, Shapiro IM. Role of cytokines in intervertebral disc degeneration: pain and disc content. Nat Rev Rheumatol 2014;10:44-56.

[9] Wuertz K, Haglund L. Inflammatory Mediators in Intervertebral Disk Degeneration and Discogenic Pain. Global Spine 2013; 3:175-184. 